\title{
ON NUMERICAL RANGES OF OPERATORS ON LOCALLY CONVEX SPACES
}

J. R. GILES, G. JOSEPH, D. O. KOEHLER AND B. SIMS

(Received 7 January 1974: revised 21 October 1974)

Communicated by J. P. O. Silberstein

Numerical range theory for linear operators on normed linear spaces and for elements of normed algebras is now firmly established and the main results of this study are conveniently presented by Bonsall and Duncan in (1971) and (1973). An extension of the spatial numerical range for a class of operators on locally convex spaces was outlined by Moore in (1969) and (1969a), and an extension of the algebra numerical range for elements of locally $m$-convex algebras was presented by Giles and Koehler (1973). It is our aim in this paper to contribute further to Moore's work by extending the concept of spatial numerical range to a wider class of operators on locally convex spaces.

\section{The spatial numerical range of quotient bounded operators}

For a separated locally convex space $X$ there exists a separating family of semi-norms $\left\{p_{\alpha}\right\}$ which generates the locally convex topology. We follow Moore in specifying that the numerical range of an operator on a locally convex space $X$ is dependent on the particular family of seminorms $\left\{p_{\alpha}\right\}$ chosen to generate the topology. We denote by $\left(X,\left\{p_{\alpha}\right\}\right)$ the linear space $X$ with a particular separating family of semi-norms $\left\{p_{\alpha}\right\}$ which generates the topology as a base. (Moore calls such a family $\left\{p_{\alpha}\right\}$ a calibration for $X$.)

Given $\left(X,\left\{p_{\alpha}\right\}\right)$ we call a linear operator $T$ on $X$ quotient bounded if for each $\alpha$ there exists a $K_{\alpha}>0$ such that

$$
p_{\alpha}(T x) \leqq K_{\alpha} p_{\alpha}(x) \text { for all } x \in X .
$$

The set of quotient bounded operators $Q\left(X,\left\{p_{\alpha}\right\}\right)$ is a subalgebra of $L(X)$ the algebra of continuous linear operators on $X$. For any given $T \in Q\left(X,\left\{p_{\alpha}\right\}\right)$ and each $\alpha$ we define 


$$
q_{\alpha}(T)=\sup \left\{p_{\alpha}(T x): p_{\alpha}(x) \leqq 1 \text { for } x \in X\right\}
$$

then $\left\{q_{\alpha}\right\}$ is a separating family of sub-multiplicative semi-norms on $Q\left(X,\left\{p_{c x}\right\}\right)$ such that $q_{\alpha}(I)=1$ for all $\alpha . Q\left(X,\left\{p_{\alpha}\right\}\right)$ with the topology generated by $\left\{q_{\alpha}\right\}$ is a unital I.m.c. algebra and we denote by $\left(Q,\left\{q_{\alpha}\right\}\right)$ the algebra $Q\left(X,\left\{p_{\alpha}\right\}\right)$ with the particular family $\left\{q_{\alpha}\right\}$ which generates the topology. (In fact this algebra is given as an example of an l.m.c. algebra by Michael (1952; page 11)).

Given $\left(X,\left\{p_{\alpha}\right\}\right)$ we call a linear operator $T$ on $X$ universally bounded if there exists $K>0$ such that, for all $\alpha$

$$
p_{\alpha}(T x) \leqq K p_{\alpha}(x) \text { for all } x \in X .
$$

The set of universally bounded operators $B\left(X,\left\{p_{\alpha}\right\}\right)$ is a subalgebra of $Q\left(X,\left\{p_{\alpha}\right\}\right)$. For any given $T \in B\left(X .\left\{p_{\alpha}\right\}\right)$ we define

$$
\|T\|=\sup \left\{p_{\alpha}(T x): p_{\alpha}(x) \leqq 1 \text { for } x \in X \text { and all } \alpha\right\} ;
$$

then $\|\cdot\|$ is a norm for $B\left(X,\left\{p_{\alpha}\right\}\right)$ and $\|T\|=\sup _{\alpha} q_{\alpha}(T)$. We denote this unital normed algebra by $(B,\|\cdot\|)$. (This is the particular class of operators to which Moore confined his attention.)

Given $\left(X,\left\{p_{c}\right\}\right)$, for each $\alpha$ let $N_{\alpha x}$ denote the null space of $p_{\alpha}$ and $X_{\alpha}$ the quotient space $X / N_{w}$. For each $\alpha$, consider the natural mapping $x \rightarrow x_{c} \equiv$ $x+N_{\alpha}$ of $X$ onto $X_{\alpha}$. It is clear that for each $\alpha, X_{\alpha}$ is a normed linear space with norm defined by $\left\|x_{c}\right\|_{\alpha x}=p_{a x}(x)$. For each $\alpha$, consider the algebra homomorphism $T \rightarrow T^{\alpha}$ of $Q\left(X,\left\{p_{\alpha}\right\}\right)$ into $B\left(X_{\alpha}\right)$ defined by $T^{\alpha} x_{\alpha}=(T x)_{\alpha}$. Since $T\left(N_{\alpha}\right) \subseteq N_{\alpha}$ for every $\alpha$, these operators $T^{\alpha}$ are well defined. But also, for each $\alpha, B\left(X_{\alpha}\right)$ is a unital normed algebra and we have for the operator norm on $B\left(X_{x}\right)$ that

$$
\begin{aligned}
\left\|T^{\alpha}\right\|_{\alpha x} \equiv \sup & \left\{\left\|T^{\alpha x} x_{\alpha x}\right\|_{\alpha x}:\left\|x_{\alpha x}\right\|_{\alpha} \leqq 1 \text { for } x_{\alpha} \in X_{\alpha}\right\} \\
& =\sup \left\{p_{\alpha}(T x): p_{\alpha}(x) \leqq 1 \text { for } x \in X\right\}=q_{\alpha}(T) .
\end{aligned}
$$

We now proceed to a definition of numerical range for quotient bounded operators.

For a normed linear space $(X,\|\cdot\|)$ we define the sets,

$$
S \equiv\{x \in X:\|x\|=1\}
$$

for each $x \in S \quad D(x) \equiv\left\{f \in X^{\prime}: f(x)=1\right.$ and $\left.\|f\|=1\right\}$, and

$$
\Pi \equiv\left\{(x, f) \in X \times X^{\prime}: x \in S \text { and } f \in D(x)\right\} .
$$

For $T \in B(X)$ we define the spatial numerical range of $T$ as the set

$$
V(X,\|\cdot\| ; T) \equiv\{f(T x):(x, f) \in \Pi\} .
$$

For each $T \in B(X)$ we have that $V(X,\|\cdot\| ; T)$ is a bounded subset of the complex numbers and is contained in the disc with centre 0 and radius $\|T\|$. 
For a separated locally convex space $X$, given $\left(X,\left\{p_{\alpha}\right\}\right)$, for each $\alpha$ consider the normed linear space $\left(\tilde{X}_{\alpha},\|\cdot\|_{\alpha}\right)$ the completion of $\left(X_{\alpha},\|\cdot\|_{\alpha}\right)$. For any given $T \in Q\left(X,\left\{p_{\alpha}\right\}\right)$ and $\alpha$, the operator $T^{\alpha}$ on $\left(X_{\alpha}\|\cdot\|_{\alpha}\right)$ has a unique continuous linear extension $\tilde{T}^{\alpha}$ on $\left(\tilde{X}_{\alpha},\|\cdot\|_{\alpha}\right)$. We define the spatial numerical range of $T$ as the set

$$
V\left(X,\left\{p_{\alpha}\right\} ; T\right) \equiv \bigcup_{\alpha} V\left(\tilde{X}_{\alpha},\|\cdot\|_{\alpha} ; \tilde{T}^{\alpha}\right) .
$$

Of course, $V\left(X,\left\{p_{\alpha}\right\} ; T\right)$ is not necessarily bounded for each $T \in Q\left(X,\left\{p_{\alpha}\right\}\right)$. But it is clear that this numerical range has the usual numerical range properties:

for $T \in Q\left(X,\left\{p_{\alpha}\right\}\right)$ and complex $\lambda, \mu$

$$
V\left(X,\left\{p_{\alpha}\right\} ; \lambda T+\mu\right)=\lambda V\left(X,\left\{p_{k}\right\} ; T\right)+\mu,
$$

and for $T, S \in Q\left(X,\left\{p_{\alpha}\right\}\right)$

$$
V\left(X,\left\{p_{c k}\right\} ; T+S\right) \subseteq V\left(X,\left\{p_{c}\right\} ; T\right)+V\left(X,\left\{p_{\alpha}\right\} ; S\right) .
$$

Given $\left(X,\left\{p_{\alpha}\right\}\right)$, for each $\alpha$ consider the semi-normed space $\left(X, p_{\alpha}\right)$ and define the sets

$$
\text { - } S_{\alpha} \equiv\left\{x \in X: p_{\alpha}(x)=1\right\}
$$

for each $x \in S_{\alpha}$

$$
D_{\alpha}(x) \equiv\left\{f \in X^{\prime}: f(x)=1 \text { and }|f(y)| \leqq p_{\alpha}(y) \quad \text { for all } y \in X\right\} .
$$

and

$$
\Pi_{\alpha} \equiv\left\{(x, f) \in X \times X^{\prime}: x \in S_{\alpha} \quad \text { and } \quad f \in D_{\alpha}(x)\right\} .
$$

For each $\alpha$ and $x \in S_{\alpha}$, consider the mapping $f \rightarrow f_{\alpha}$ of $D_{\alpha}(x)$ onto $D\left(x_{*}\right)$ defined by $f_{a}\left(y_{x}\right)=f(y)$. For every $f \in D_{a}(x)$. since $N_{a} \subseteq \operatorname{ker} f$ the linear functionals $f_{\alpha}$ are well defined and we have that $f_{\alpha}\left(x_{\alpha}\right)=f(x)=1$ and $\left|f_{\alpha}\left(y_{\alpha}\right)\right|=$ $|f(y)| \leqq p_{\alpha}(y)=\left\|y_{\alpha}\right\|_{\alpha}$ for all $y \in X$. It follows that for $T \in Q\left(X .\left\{p_{\alpha}\right\}\right), f(T x) \in$ $V\left(X,\left\{p_{c}\right\} ; T\right)$ for all $\alpha$ and $(x, f) \in \Pi_{\alpha}$.

\section{The numerical range and the spectrum}

In a Banach space, the closure of the spatial numerical range of an operator contains its spectrum. We now show that this relation holds for all quotient bounded operators on a complete locally convex space.

Lemma 1. Let $X$ be a complete separated locally convex space. Given $\left(X,\left\{p_{\alpha}\right\}\right)$ and $T \in Q\left(X,\left\{p_{\alpha}\right\}\right), T$ is invertible in $Q\left(X,\left\{p_{\alpha}\right\}\right)$ if and only if $\tilde{T}^{\alpha}$ is invertible in $B\left(\tilde{X}_{\alpha}\right)$ for all $\alpha$. 
Proof. If $T$ has an inverse $T^{\cdots 1} \in Q\left(X,\left\{p_{c}\right\}\right)$ then $\tilde{T}^{\prime \prime}$ has an inverse $\tilde{T}^{-1 \alpha} \in B\left(\tilde{X}_{x}\right)$ for each $\alpha$.

Conversely, suppose that for each $\alpha . \tilde{T}^{\alpha}$ has an inverse in $B\left(\tilde{X}_{\alpha r}\right)$. Then for each $\alpha$ there exists an $m_{x}>0$ such that

$$
m_{\alpha} p_{\alpha}(x) \leqq p_{\alpha}(T x) \text { for all } x \in X .
$$

Since $\left\{p_{\alpha}\right\}$ is a separating family, *implies that $T$ is one-to-one.

$T$ has a closed range. For any net $\left\{x_{\delta}\right\}$ such that $\left\{T x_{\delta}\right\}$ is convergent to $y \in X$, we have from* that $\left\{x_{8}\right\}$ is Cauchy in $X$. But since $X$ is complete there exists an $x \in X$ such that $\left\{x_{\delta}\right\}$ is convergent to $x$ and since $T$ is continuous $y=T x$.

$T$ also has a dense range. Consider $y \in X$ and any basic neighbourhood of $y, U \equiv\left\{x \in X: p_{\alpha}(y-x)<\varepsilon\right\}$ for any given $\alpha$ and $\varepsilon>0$. Since $\tilde{T}^{\alpha}$ is onto and continuous on $\tilde{X}_{\alpha}$ there exists an $x \in X$ such that $\left\|y_{\alpha}-T^{\alpha} x_{\alpha}\right\|_{\alpha}<\varepsilon$; that is, $p_{\alpha}(y-T x)<\varepsilon$ and so $T x \in U$.

We conclude that $T$ has an inverse $T^{-1}$ and from * that $T^{-1} \in Q\left(X,\left\{p_{\alpha}\right\}\right)$.

Corollary. Let $X$ be a complete separated locally convex space. Given $\left(X,\left\{p_{\alpha}\right\}\right)$ and $T \in Q\left(X,\left\{p_{\alpha}\right\}\right)$

$$
\sigma(Q ; T)=\bigcup_{\alpha} \sigma\left(X_{\alpha} ; T^{\alpha}\right)=\bigcup_{\alpha} \sigma\left(\tilde{X}_{\alpha} ; \tilde{T}^{\alpha}\right) .
$$

Proof. It is clear that even when $X$ is not complete,

$$
\sigma(Q ; T) \supseteq \bigcup_{\alpha} \sigma\left(X_{\alpha} ; T^{\alpha}\right)
$$

and for every $\alpha$

$$
\sigma\left(X_{\alpha} ; T^{\alpha}\right) \supseteq \sigma\left(\tilde{X}_{\alpha} ; \tilde{T}^{\alpha}\right) .
$$

But from the lemma we have directly that

$$
\sigma(Q ; T)=\bigcup_{\kappa} \sigma\left(\bar{X}_{\alpha} ; \tilde{T}^{\alpha}\right) .
$$

THEOREM 1. Let $X$ be a complete separated locally convex space. Given $\left(X,\left\{p_{\alpha}\right\}\right)$, for any $T \in Q\left(X,\left\{p_{\alpha}\right\}\right)$

$$
\sigma(Q ; T) \subseteq \overline{V\left(X,\left\{p_{\alpha}\right\} ; T\right)}
$$

Proof. From the normed linear space theory see Bonsall and Duncan (1971; page 88 ) we have for each $\alpha$

$$
\sigma\left(\tilde{X}_{\alpha} ; \tilde{T}^{\alpha}\right) \subseteq \overline{V\left(\bar{X}_{c},\|\cdot\|_{\alpha} ; \tilde{T}^{\alpha}\right)}
$$

Therefore, 


$$
\begin{aligned}
& \sigma(Q ; T)=\bigcup_{\alpha} \sigma\left(\tilde{X}_{\alpha} ; \tilde{T}^{\alpha}\right) \\
& \subseteq \bigcup_{\alpha} \overline{V\left(\tilde{X}_{\alpha} ;\|\cdot\|_{\alpha} ; \tilde{T}^{\alpha}\right)} \subseteq \overline{\bigcup_{\alpha} V\left(\tilde{X}_{\alpha} ;\|\cdot\|_{\alpha} ; \tilde{T}^{\alpha}\right)}=\overline{V\left(X,\left\{p_{\alpha}\right\} ; T\right)} .
\end{aligned}
$$

\section{The relation between the spatial and algebra numerical ranges}

For a unital normed algebra the spatial numerical range of an element acting as an operator on the algebra is the algebra numerical range of the element. We show that this relation holds for elements of a unital l.m.c. algebra.

For a unital normed algebra $(A,\|\cdot\|)$ we define the set

$$
D(1) \equiv\left\{f \in A^{\prime}: f(1)=1 \text { and }\|f\|=1\right\} .
$$

For $a \in A$ we define the algebra numerical range of $a$ as the set

$$
V(A,\|\cdot\| ; a) \equiv\{f(a): f \in D(1)\} .
$$

For each $a \in A$ we have that $V(A,\|\cdot\| ; a)$ is a compact convex subset of the complex numbers and is contained in the disc with centre 0 and radius $\|a\|$. Considering the left regular representation $a \rightarrow T_{a}$ of $A$ in $B(A)$ we have that

$$
V(A,\|\cdot\| ; a)=V\left(A,\|\cdot\| ; T_{a}\right), \quad \text { (Bonsall and Duncan (1971; page 15)), }
$$

Let $A$ be a unital 1.m.c. algebra and $\left\{p_{\alpha}\right\}$ be a separating family of sub-multiplicative semi-norms which generates the topology and is such that $p_{\alpha}(1)=1$ for all $\alpha$. Given $\left(A,\left\{p_{\alpha}\right\}\right)$, we define the algebra numerical range of $a$ as the set

$$
V\left(A,\left\{p_{\alpha}\right\} ; a\right) \equiv \bigcup_{\alpha} V\left(A_{\alpha},\|\cdot\|_{\alpha} ; a_{\alpha}\right) .
$$

Considering the left regular representation $a \rightarrow T_{a}$ of $A$ in $T(A)$, since the semi-norms $p_{\alpha}$ are sub-multiplicative it follows that the image of $A$ is a subalgebra of $Q\left(A,\left\{p_{\alpha}\right\}\right)$. For any given $a \in A$ and $\alpha$, and all $x \in A$, we have

$$
(a x)_{\alpha}=\left(T_{a} x\right)_{\alpha}=\left(T_{a}\right)^{\alpha} x_{\alpha}
$$

and since the natural mapping $x \rightarrow x_{\alpha}$ is here an algebra homomorphism

$$
(a x)_{\alpha}=a_{\alpha} x_{\alpha}=T_{a_{\alpha}} x_{c},
$$

and so $\left(T_{a}\right)^{\alpha}=T_{a_{\alpha}}$.

Therefore, from the invariance of the normed algebra numerical ranges under completions (Bonsall and Duncan (1971; page 16)) we have for each $\alpha$,

$$
\begin{aligned}
V\left(A_{\alpha},\|\cdot\|_{\alpha} ; a_{\alpha}\right) & =V\left(\tilde{A}_{\alpha},\|\cdot\|_{\alpha} ; a_{\alpha}\right) \\
& =V\left(\tilde{A}_{\alpha},\|\cdot\|_{\alpha} ; T_{a_{\alpha}}\right)=V\left(\tilde{A}_{\alpha},\|\cdot\|_{\alpha} ;\left(\tilde{T}_{a}\right)^{\alpha}\right),
\end{aligned}
$$

and so, as in the normed case, we have 


$$
V\left(A,\left\{p_{\alpha}\right\} ; a\right)=V\left(A,\left\{p_{\alpha}\right\} ; T_{a}\right) .
$$

For a normed linear space $(X,\|\cdot\|)$, the closed convex hull of the spatial numerical range of a continuous linear operator on $X$ is the algebra numerical range of the operator as an element of the unital normed algebra of operators $B(X)$. We now show that for a separated locally convex space $X$, given $\left(X,\left\{p_{\alpha}\right\}\right)$ there is a similar relation between the spatial numerical range of a quotient bounded operator and the algebra numerical range of the operators as an element of the unital l.m.c. algebra $\left(Q,\left\{q_{\alpha}\right\}\right)$.

Given $\left(Q,\left\{q_{\alpha}\right\}\right)$, for each $\alpha$ let $M_{\alpha}$ denote the null space of $q_{\alpha x}$ and $Q_{\alpha}$ denote the quotient algebra $Q / M_{\alpha}$. For each $\alpha$, consider the natural mapping $T \rightarrow T_{\alpha} \equiv T+M_{\alpha}$ of $Q$ onto $Q_{\alpha}$. It is clear that for each $\alpha, Q_{\alpha}$ is a unital normed algebra with norm defined by $\left\|T_{\alpha}\right\|_{\alpha}=q_{\alpha}(T)$.

For each $\alpha$, we need to examine the relation between the unital normed algebras $Q_{\alpha}$ and $B\left(\tilde{X}_{\alpha}\right)$.

Lemma 2. Let $X$ be a separated locally convex space. Given $\left(X,\left\{p_{\alpha}\right\}\right)$, we have that for each $\alpha, Q_{\alpha}$ is isometrically isomorphic to a unital subalgebra of $B\left(\tilde{X}_{\alpha}\right)$.

Proof. For any given $\alpha$, consider the mapping $T_{\alpha} \rightarrow \tilde{T}^{\alpha}$ of $Q_{\alpha}$ into $B\left(\bar{X}_{\alpha}\right)$. The mapping is well defined and is an isometry. For $T, S \in Q\left(X,\left\{p_{\alpha}\right\}\right)$,

$$
\left\|T_{\alpha}-S_{\alpha \alpha}\right\|_{\alpha}=\left\|(T-S)_{\alpha}\right\|_{\alpha}=q_{\alpha}(T-S)=\left\|(T-S)^{\alpha}\right\|_{\alpha}=\left\|T^{\alpha}-S^{\alpha}\right\|_{\alpha} .
$$

The mapping is an algebra homomorphism. The mappings $T \rightarrow T_{\alpha}$ of $Q$ onto $Q_{\alpha}$ and $T \rightarrow \tilde{T}^{\alpha}$ of $Q$ into $B\left(\tilde{X}_{\alpha}\right)$ are algebra homomorphisms, so it follows from the mapping $T_{\alpha} \rightarrow \tilde{T}^{\alpha}$ of $Q_{\alpha}$ into $B\left(\tilde{X}_{\alpha}\right)$ being well defined, that it is also an algebra homomorphism.

Corollary. Let $X$ be a separated locally convex space. Given $\left(X,\left\{p_{\alpha}\right\}\right)$, for any $T \in\left(Q,\left\{q_{\alpha}\right\}\right)$

$$
V\left(Q,\left\{q_{\alpha}\right\} ; T\right)=\bigcup_{\alpha} V\left(B\left(\tilde{X}_{\alpha}\right),\|\cdot\|_{\alpha} ; \tilde{T}^{\alpha}\right) .
$$

Proof. We have

$$
V\left(Q,\left\{q_{\alpha}\right\} ; T\right)=\bigcup_{\alpha} V\left(Q_{\alpha},\|\cdot\|_{\alpha} ; T_{\alpha}\right) .
$$

But from the lemma and the invariance of the normed algebra numerical range under unital subalgebras (Bonsall and Duncan (1971; page 16)) we have for each $\alpha$,

$$
V\left(Q_{\alpha},\|\cdot\|_{\alpha} ; T_{\alpha}\right)=V\left(B\left(\tilde{X}_{\alpha}\right),\|\cdot\|_{\alpha} ; \tilde{T}^{\alpha}\right)
$$


THEOREM 2. Let $X$ be a separated locally convex space. Given $\left(X,\left\{p_{c}\right\}\right)$, for any $T \in Q\left(X,\left\{p_{c}\right\}\right)$ we have

$$
V\left(X .\left\{p_{n}\right\}: T\right) \subseteq V\left(Q .\left\{q_{n}\right\}: T\right) \subseteq \overline{c o} V\left(X .\left\{p_{n}\right\} ; T\right) .
$$

and when $\left\{q_{\alpha}\right\}$ is a directed family.

$$
\overline{\operatorname{co}} V\left(X,\left\{p_{\alpha}\right\} ; T\right)=\overline{V\left(Q,\left\{q_{\alpha}\right\} ; T\right)} .
$$

Proof. We have established the characterisation

$$
V\left(Q .\left\{q_{v}\right\}: T\right)=\bigcup_{\iota} V\left(B\left(\tilde{X}_{c}\right),\|\cdot\|_{\alpha}: \tilde{T}^{\alpha}\right) .
$$

But from the normed case (Bonsall and Duncan (1971; page 84)) we have, for each $\alpha$,

So

$$
V\left(B\left(\tilde{X}_{a}\right) .\|\cdot\|_{a} ; \tilde{T}^{(\alpha)}\right)=\overline{\operatorname{co}} V\left(\tilde{X}_{w}\|\cdot\|_{a} ; \tilde{T}^{\alpha}\right) .
$$

$$
V\left(Q,\left\{q_{\alpha}\right\}: T\right) \supseteq \bigcup_{*} V\left(\tilde{X}_{a}\|\cdot\|_{\alpha}: \tilde{T}^{\alpha}\right)=V\left(X .\left\{p_{n}\right\} ; T\right) .
$$

But also

$$
\begin{aligned}
& V\left(Q,\left\{q_{\alpha}\right\} ; T\right)=\bigcup_{*} \overline{\operatorname{co}} V\left(\tilde{X}_{\alpha} \mid \cdot \|_{\alpha} ; \tilde{T}^{\alpha}\right)
\end{aligned}
$$

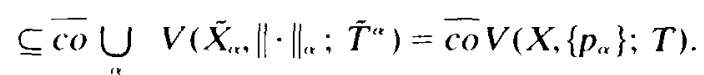

When $\left\{q_{\alpha}\right\}$ is a directed family for the I.m.c. algebra $\left(Q,\left\{q_{\alpha}\right\}\right)$ we have that $V\left(Q,\left\{q_{i}\right\} ; T\right)$ is convex and so

$$
\overline{\operatorname{co}} V\left(X,\left\{p_{. x}\right\} ; T\right)=\overline{V\left(Q,\left\{q_{*}\right\}: T\right)}
$$

\section{Operators with bounded numerical range}

We are now in a position to discuss boundedness of the spatial numerical ranges of quotient bounded operators and to characterise classes of these operators by boundedness of their numerical ranges and spectra.

Using Theorem 2 which relates the spatial and algebra numerical ranges we are able to apply boundedness results previously established for algebra numerical ranges.

TheOrem 3. Let $X$ be a separated locally convex space. Given $\left(X,\left\{p_{a}\right\}\right)$,

$$
B\left(X,\left\{p_{\alpha}\right\}\right)=\left\{T \in Q\left(X,\left\{p_{\alpha}\right\}\right): V\left(X,\left\{p_{\alpha}\right\}: T\right) \text { is bounded }\right\} .
$$

Proof. For $T \in Q\left(X .\left\{p_{a}\right\}\right)$, if $V\left(X .\left\{p_{a}\right\} ; T\right)$ is bounded then by Theorem 2, $V\left(Q,\left\{q_{\alpha}\right\} ; T\right)$ is bounded and so by Giles and Koehler (1973; page 83) we have that $\sup q_{\alpha}(T)<\infty$ which implies that $T \in B\left(X,\left\{p_{\alpha}\right\}\right)$. 
Conversely, it follows from Giles and Koehler (1973; page 85) that if $T \in B\left(X,\left\{p_{\alpha}\right\}\right)$ then $V\left(Q,\left\{q_{\alpha}\right\} ; T\right)$ is bounded and so by Theorem 2, $V\left(X,\left\{p_{\alpha}\right\} ; T\right)$ is bounded.

It follows that, if $T \in Q\left(X,\left\{p_{c}\right\}\right) \backslash B\left(X,\left\{p_{c}\right\}\right)$ then $V\left(X,\left\{p_{c}\right\} ; T\right)$ is unbounded. Nevertheless, we show that, when $X$ is complete. for any $T \in$ $Q\left(X,\left\{p_{\alpha}\right\}\right)$ with bounded spectrum $\sigma(Q ; T)$ it is possible to find a family of semi-norms $\left\{p_{\prime \prime}^{\prime}\right\}$ generating the topology of $X$ such that $T \in Q\left(X,\left\{p_{c}^{\prime}\right\}\right)$ and the numerical range $V\left(X,\left\{p_{i}^{\prime}\right\}: T\right)$ is bounded.

For a locally convex space, given $\left(X,\left\{p_{c}\right\}\right)$ we say that a family of semi-norms $\left\{\boldsymbol{p}_{\text {, }}^{\prime}\right\}$ generating the topology of $X$ is quotient preserving if it has the same indexing and for each $\alpha$ there exist $m_{c}, M_{c}>0$ such that

$$
m_{a p} p_{w}(x) \leqq p_{u r}^{\prime}(x) \leqq M_{c} p_{a}(x) \text { for all } x \in X .
$$

It is clear that $Q\left(X,\left\{p_{c}\right\}\right)=Q\left(X .\left\{p_{c}^{\prime}\right\}\right)$.

THEOREM 4. Let $X$ be a complete separated locally convex space. Given $\left(X,\left\{p_{c}\right\}\right)$, if $T \in Q\left(X,\left\{p_{c}\right\}\right)$ and $\sigma(Q: T)$ is bounded then there exists a quotient preserving family of semi-norms $\left\{p_{c}^{\prime}\right\}$ generating the topology of $X$ such that

(i) $T \in B\left(X .\left\{p_{i k}^{\prime}\right\}\right)$, and

(ii) the spectral radius, $\rho(Q ; T)$

$=\inf \left\{\|T\|^{\prime}\right.$ : all quotient preserving families $\left\{p_{c}^{\prime}\right\}$

generating the topology of $X\}$.

Proof. We have from the Corollary to Lemma 1 that

and so

$$
\sigma(Q ; T)=\bigcup_{*} \sigma\left(\tilde{X}_{c} ; \tilde{T}^{\alpha}\right)
$$

$$
\rho(Q ; T)=\sup \rho\left(\tilde{X}_{a} ; \tilde{T}^{*}\right) .
$$

For each $\alpha$ consider $\left(\tilde{X}_{\alpha},\|\cdot\|_{\alpha}\right)$. We have from Holmes (1968; page 164) that

$$
\rho\left(\tilde{X}_{\alpha} ; \tilde{T}^{\alpha}\right)=\inf \left\{\left\|\tilde{T}^{\alpha}\right\|_{\alpha}^{\prime}: \text { for all equivalent norms }\|\cdot\|_{\alpha}^{\prime} \text { for } \tilde{X}_{\alpha}\right\} .
$$

Therefore, given $\varepsilon>0$, for each $\alpha$ there exists an equivalent norm $\|\cdot\|_{\alpha}^{\prime}$ on $\tilde{X}_{\alpha}$ such that $\dagger$

$$
\rho\left(\tilde{X}_{u}: \tilde{T}^{\alpha}\right) \leqq\left\|\tilde{T}^{\alpha}\right\|_{\alpha}^{\prime} \leqq \rho\left(\tilde{X}_{\alpha} ; \tilde{T}^{\alpha}\right)+\varepsilon .
$$

For each $\alpha$ such a norm $\|\cdot\|_{\alpha}^{\prime}$ on $\tilde{X}_{\alpha}$ induces a semi-norm $p_{\alpha}^{\prime}$ on $X$ defined by

$$
p_{c x}^{\prime}(x)=\left\|x_{a x}\right\|_{i x}^{\prime}
$$

and we have that there exist $m_{\alpha}, M_{\alpha}>0$ such that 


$$
m_{\alpha x} p_{c x}(x) \leqq p_{c x}^{\prime}(x) \leqq M_{\alpha} p_{\alpha}(x) \text { for all } x \in X .
$$

It is clear therefore that $\left\{p_{\alpha}^{\prime}\right\}$ is a quotient preserving family of semi-norms generating the topology on $X$. Now since $\sigma(Q ; T)$ is bounded it follows from ${ }^{\dagger}$ that $\left\{\left\|\tilde{T}^{\alpha}\right\|_{\alpha}^{\prime}\right\}$ is bounded and so

$$
V\left(X,\left\{p_{\alpha}^{\prime}\right\} ; T\right)=\bigcup_{\alpha} V\left(\tilde{X}_{\alpha},\|\cdot\|_{\alpha}^{\prime} ; \tilde{T}^{\alpha}\right)
$$

is bounded and $T \in B\left(X,\left\{p_{\alpha}^{\prime}\right\}\right)$ which is result (i).

It also follows from ${ }^{\dagger}$ that

$$
\begin{aligned}
\rho(Q ; T) & =\sup _{\alpha} \rho\left(\tilde{X}_{\alpha} ; \tilde{T}^{\alpha}\right) \\
& \leqq\|T\|^{\prime}=\sup _{\alpha}\left\|\tilde{T}^{\alpha}\right\|_{\alpha}^{\prime} \\
& \leqq \sup _{\alpha} \rho\left(\tilde{X}_{\alpha} ; \tilde{T}^{\alpha}\right)+\varepsilon=\rho(Q ; T)+\varepsilon
\end{aligned}
$$

and so we establish (ii).

We can deduce directly the following corollary which is similar to that of Holmes (1968; page 165).

For a locally convex space $X$, given $\left(X,\left\{p_{\alpha}\right\}\right)$ we say that $T \in B\left(X,\left\{p_{\alpha}\right\}\right)$ is a strict contraction if $\|T\|<1$.

Corollary. Let $X$ be a complete separated locally convex space. Given $\left(X,\left\{p_{\alpha}\right\}\right)$, if $T \in Q\left(X,\left\{p_{\alpha}\right\}\right)$ then there exists a quotient preserving family of semi-norms $\left\{p_{\alpha}^{\prime}\right\}$ generating the topology of $X$ for which $T$ is a strict contraction if and only if $\rho(Q ; T)<1$.

It should be observed that

1. there are quotient bounded operators with unbounded spectrum, and

2. there are continuous linear operators with bounded spectrum but which are not quotient bounded.

EXAMPLES.

1. Consider the linear space $X$ of complex mappings on $\mathbf{C}$, with the topology generated by the family of semi-norms $\left\{p_{F}\right\}$ where

$$
p_{F}(f)=\max \{|f(x)|: x \in F \text { a finite subset of } C\} .
$$

Consider the linear operator $T$ on $X$ defined by

$$
(T f)(x)=x . f(x) \text { for all } x \in \mathbf{C} .
$$

Now for every $x \in \mathbf{C}$, 


$$
\begin{aligned}
p_{\{x\}}(T f) & =|T f(x)|=|x||f(x)| \\
& =|x| p_{\{x\}}(f),
\end{aligned}
$$

and so $T \in Q\left(X,\left\{p_{F}\right\}\right)$.

Suppose that $\lambda \notin \sigma(Q ; T)$; that is, $T-\lambda I$ has an inverse $S \in Q\left(X,\left\{p_{F}\right\}\right)$. Then

$$
(T-\lambda I)(S f)=f \text { for all } f \in X
$$

so

$$
(x-\lambda)(S f)(x)=f(x) \text { for all } f \in X \text { and } x \in \mathbf{C} .
$$

Choose $f$ to be a constant mapping, $f(x)=k \neq 0$ for all $x \in \mathbf{C}$, then

$$
(x-\lambda)(S f)(x)=k \text { for all } x \in \mathbf{C},
$$

but this gives a contradiction when $x=\lambda$. We conclude that $\sigma(Q ; T)=\mathbf{C}$.

2. Consider the linear space of complex sequences with topology generated by the family of semi-norms $\left\{p_{n}\right\}$ where

$$
p_{n}\left(\lambda_{1}, \lambda_{2}, \cdots\right)=\sup \left\{\left|\lambda_{k}\right|: k \in\{1, \cdots, n\}\right\} .
$$

Consider the operator $T$ on $X$ defined by

$$
T\left(\lambda_{1}, \lambda_{2}, \lambda_{3}, \cdots\right)=\left(\lambda_{2}, \lambda_{1}, \lambda_{3}, \cdots\right) .
$$

Then $T$ is linear and continuous. Clearly $T \notin Q\left(X,\left\{p_{\alpha}\right\}\right)$.

Now \pm 1 are eigenvalues of $T$.

For $\alpha \neq \pm 1$ we can define the operator $S$ on $X$ by

$$
S\left(\lambda_{1}, \lambda_{2}, \lambda_{3}, \cdots\right)=\left(\frac{\alpha \lambda_{1}+\lambda_{2}}{\alpha^{2}-1}, \frac{\lambda_{1}+\alpha \lambda_{2}}{\alpha^{2}-1}, \frac{\lambda_{3}}{\alpha-1}, \cdots\right) .
$$

It is simply verified that $S$ is an inverse of $\alpha I-T$ and that $S \in L(X)$. Therefore $\sigma(X ; T)=\{-1,+1\}$.

\section{Hermitian operators}

Numerical range theory enables an extension of the notion of hermitian operators to operators on normed linear spaces. We now define and examine properties of hermitian operators on separated locally convex spaces.

For a separated locally convex space $X$, given $\left(X,\left\{p_{\alpha}\right\}\right)$ we say that $T \in Q\left(X,\left\{p_{\alpha}\right\}\right)$ is hermitian if $V\left(X,\left\{p_{\alpha}\right\} ; T\right) \subseteq \mathbf{R}$.

For hermitian operators with bounded spectrum we have the following result. 
THEOREM 5. For a separated locally convex space $X$, given $\left(X,\left\{p_{\alpha}\right\}\right)$, if $T \in Q\left(X,\left\{p_{\alpha}\right\}\right)$ is hermitian and $\sigma(Q ; T)$ is bounded then $T \in B\left(X,\left\{p_{\alpha x}\right\}\right)$.

Proof. By Theorem 2 we have that

$$
V\left(Q,\left\{q_{\alpha}\right\} ; T\right) \subseteq \overline{\operatorname{co}} V\left(X,\left\{p_{c}\right\} ; T\right)
$$

so $V\left(Q,\left\{q_{r}\right\} ; T\right) \subseteq \mathbf{R}$. But

$$
V\left(Q,\left\{q_{\alpha}\right\} ; T\right)=\bigcup_{\sim} V\left(Q_{*},\|\cdot\|_{\infty} ; T_{\alpha}\right),
$$

so for each $\alpha, V\left(Q_{\ldots}\left\|_{\cdot}\right\|_{\alpha}: T_{c}\right) \subseteq \mathbf{R}$; that is, $T_{a}$ is an hermitian element of $\left(Q_{\alpha},\|\cdot\|_{\alpha}\right)$. However, by Sinclair's Theorem (Bonsall and Duncan (1971; page 54))

$$
\rho\left(Q_{\alpha c} ; T_{\alpha}\right)=\left\|T_{c \alpha}\right\|_{\alpha}=q_{\alpha}(T),
$$

but $\sigma(Q ; T) \supseteq \cup_{\alpha} \sigma\left(Q_{\alpha} ; T_{\alpha}\right)$ so $\rho(Q ; T) \geqq \sup _{\alpha} \rho\left(Q_{\alpha} ; T_{\alpha}\right)=\sup _{\alpha} q_{\alpha}(T)$.

We conclude that $\sup _{\alpha} q_{\alpha}(T)<x$; that is, $T \in B\left(X,\left\{p_{\alpha}\right\}\right)$.

It should be noted that there are hermitian quotient bounded operators with unbounded spectrum. Our example uses the following lemma which may also be deduced from Proposition A4 of Michael (1952; page 70).

Lemma 3. Let $X$ be a complete separated locally convex space. Given $\left(X,\left\{p_{\alpha}\right\}\right), Q\left(X,\left\{p_{\alpha}\right\}\right)$ is also complete.

Proof. Let $\left\{T_{\delta}\right\}$ be a Cauchy net in $\left(Q,\left\{q_{\alpha}\right\}\right)$. We deduce that for each $x \in X,\left\{T_{\delta} x\right\}$ is a Cauchy net in $\left(X,\left\{p_{\alpha}\right\}\right)$. But since $X$ is complete we can define the operator $T$ on $X$ by

$$
T x=\lim _{\delta} T_{\delta} x .
$$

Clearly $T$ is linear. But also for each $\alpha,\left\{q_{\alpha}\left(T_{\delta}\right)\right\}$ is a convergent net of real numbers. Therefore, for each $\alpha$ and all $x \in X$

$$
\begin{aligned}
p_{\alpha}(T x) & =p_{\alpha}\left(\lim _{\delta} T_{\delta} x\right) \\
& =\lim _{\delta} p_{\alpha}\left(T_{\delta} x\right) \leqq \lim _{\delta} q_{\alpha}\left(T_{\delta}\right) p_{\alpha}(x) .
\end{aligned}
$$

But this implies that $T \in Q\left(X,\left\{p_{c}\right\}\right)$.

For each $\alpha$, given $\varepsilon>0$ there exists a $\delta_{0}$ such that

$$
q_{n}\left(T_{\delta}-T_{\delta^{\prime}}\right)<\varepsilon \text { for all } \delta, \delta^{\prime} \geqq \delta_{01} .
$$

But then for all $x \in X$ where $p_{\alpha}(x) \leqq 1$ we have 


$$
p_{x}\left(T_{s} x-T_{\delta} x\right)<\varepsilon \text { for all } \delta, \delta^{\prime} \geqq \delta_{0}
$$

which implies that

$$
\begin{aligned}
\lim _{\delta} p_{a x}\left(T_{\delta} x-T_{\delta}^{\prime} x\right) & =p_{c k}\left(T_{\delta} x-T x\right) \\
& \leqq \varepsilon \text { for all } \delta \geqq \delta_{0} .
\end{aligned}
$$

Therefore

$$
q_{\mathrm{c}}\left(T_{\delta}-T\right) \leqq \varepsilon \text { for all } \delta \geqq \delta_{0},
$$

and we conclude that $\left(Q,\left\{q_{1 .}\right\}\right)$ is complete.

EXAMPI.E.

Consider the complete l.m.c. algebra $X$ of all complex mappings on $\mathbf{R}$. with topology generated by the family of semi-norms $\left\{p_{F}\right\}$ where

$$
p_{F}(f)=\max \{|f(x)|: x \in F \text { a finite subset of } \mathbf{R}\} .
$$

Consider the linear operator $T$ on $X$ defined by

$$
(T f)(x)=x, f(x) \text { for all } x \in \mathbf{R} \text {. }
$$

As with Example 1 above it is shown similarly that $T \in Q\left(X,\left\{p_{F}\right\}\right)$ and $\sigma(Q ; T) \subseteq \mathbf{R}$. Now $T f=j . f$ where $j$ is the identity mapping on $\mathbf{R}$. Since $Q\left(X,\left\{p_{F}\right\}\right)$ is complete the operator $\exp T \in Q\left(X,\left\{p_{F}\right\}\right)$. For $\lambda \in \mathbf{R}$ we have

$$
(\exp i \lambda T)(f)=(\exp i \lambda j) \cdot f
$$

and for any $x \in \mathbf{R}$

$$
p_{\{x\}}((\exp i \lambda T) f)=|\exp i \lambda x| p_{\{x\}}(f)
$$

so

$$
p_{F}((\exp i \lambda T) f)=p_{F}(f),
$$

and from Bonsall and Duncan (1971; page 46) we conclude that $T$ is hermitian.

The following theorem applies our theory to characterise hermitian quotient bounded operators on unital commutative $b *$-algebras.

Theorem 6. Let $A$ be a unital commutative $b^{*}$-algebra. Given a characterising $\left(A,\left\{p_{\alpha}\right\}\right), T \in Q\left(A,\left\{p_{a}\right\}\right)$ is hermitian if and only if there exists a hermitian element $y \in A$ such that

$$
T(x)=y \cdot x \text { for all } x \in A \text {. }
$$

Proof. If $T$ is hermitian on $A$ then for each $\alpha$. $\tilde{T}^{\alpha}$ is hermitian on $\tilde{A}_{\alpha}$. But since $A$ is a $b^{*}$-algebra, $\tilde{A}_{\alpha}$ is a $B^{*}$-algebra Giles and Koehler (1973; page 88). So $\tilde{T}^{\alpha}$ is an hermitian operator on a commutative $B^{*}$-algebra $\tilde{A}_{\alpha}$. By the 
Gelfand-Naimark Representation Theorem for commutative $B^{*}$-algebras we may take $\tilde{T}^{\alpha}$ as an hermitian operator on $\mathscr{C}\left(\mathcal{M}_{\alpha}\right)$ where $\mu_{\alpha}$ is the maximal ideal space of $\tilde{A}_{\alpha}$. Now Lumer has characterised such operators as multiplication by real continuous mappings (Bonsall and Duncan (1973; page 91)). So for each $\alpha$ there exists a $y_{\alpha} \in \tilde{A}_{\alpha}$ such that $y_{\alpha}$ is hermitian and $\tilde{T}^{\alpha} x_{\alpha}=y_{\alpha} x_{\alpha}$ for all $x_{\alpha} \in \bar{A}_{\alpha}$. Now

$$
\begin{aligned}
& \tilde{T}^{\alpha}\left(1_{\alpha}\right)=y_{\alpha} \cdot 1_{\alpha}=y_{\alpha} \text { and } \\
& \tilde{T}^{\alpha}\left(x_{\alpha}\right)=y_{\alpha} \cdot x_{\alpha}=\tilde{T}^{\alpha}\left(1_{\alpha}\right) x_{\alpha}=(T(1) x)_{\alpha} .
\end{aligned}
$$

So $T x=T(1) x$ for all $x \in A$. Since $y_{\alpha}$ is hermitian for each $\alpha$ so is the element $T(1) \in A$.

Conversely, if $T(x)=y . x$ where $y$ is hermitian then $\tilde{T}^{\alpha} x_{\alpha}=y_{\alpha} \cdot x_{x}$ for every $\alpha$, so that $\tilde{T}^{\text {a }}$ is multiplication by an hermitian element on a commutative $B^{*}$-algebra $\tilde{A}_{\alpha}$. By the Lumer characterisation, $\tilde{T}^{\alpha}$ is hermitian on $\tilde{A}_{\alpha}$ and so $T$ is hermitian on $A$.

As a special case we have the following result which can also be proved simply and independently of Lumer's characterisation.

COROLLARY. Let $\Omega$ be a locally compact Hausdorff space and $\mathscr{C}(\Omega)$ be the algebra of complex continuous mappings on $\Omega$, with the compact-open topology. A quotient bounded operator $T$ on $\mathscr{C}(\Omega)$ is hermitian if and only if there exists a real continuous mapping $h$ on $\Omega$ such that

$$
T(f)=h . f \text { for all } f \in \mathscr{C}(\Omega) \text {. }
$$

\section{Representation of $b^{*}$-algebras}

The Gelfand-Naimark Representation Theorem for $B^{*}$-algebras states that a $B^{*}$-algebra is isometrically * isomorphic to a closed self-adjoint subalgebra of operators on a Hilbert space. We now extend this result to give a representation of $b^{*}$-algebras.

We firstly establish the character of the algebra of quotient bounded operators on a product of Hilbert spaces as a $b^{*}$-algebra.

THEOREM 7. Given a family $\left\{\left(H_{\alpha},\|\cdot\|_{\alpha}\right)\right\}$ of Hilbert spaces, the algebra $Q\left(\Pi H_{c},\left\{\|\cdot\|_{\alpha}\right\}\right)$ of quotient bounded operators on the product space $\Pi H_{\alpha}$ is a $b^{*}$-algebra.

Proof. It is clear that $I H_{\alpha}$ is complete and so from Lemma 3 we have that $Q\left(\Pi H_{\alpha},\left\{\|\cdot\|_{\alpha}\right\}\right)$ is also complete. Given $T \in Q\left(\Pi H_{\alpha},\left\{\|\cdot\|_{\alpha}\right\}\right)$, for every $\alpha$ there exists a $K_{\alpha}>0$ such that $\left\|T^{\alpha} x_{\alpha}\right\|_{\alpha} \leqq K_{\alpha}\left\|x_{\alpha}\right\|_{\alpha}$. It is clear that the *-operation on $B\left(H_{\alpha}\right)$ for each $\alpha$ induces a *operation on $Q\left(\Pi H_{\alpha},\left\{\|\cdot\|_{\alpha}\right\}\right)$ defined by 
$\left(T^{*} x\right)_{\alpha}=T^{\alpha *} x_{\alpha}$. But for each $\alpha$, since $q_{\alpha}(T)=\left\|T^{\alpha}\right\|_{\alpha}$ we have that $q_{\alpha}\left(T^{*} T\right)=q_{\alpha}(T)^{2}$.

We now show that a $b^{*}$-algebra can be represented as a closed self-adjoint subalgebra of the quotient bounded operators on a product of Hilbert spaces.

Tнеовем 8. Let $A$ be $a b^{*}$-algebra. Given $\left(A,\left\{q_{\alpha}\right\}\right)$, for each $\alpha$ there exists a Hilbert space $\left(H_{\alpha},\|\cdot\|_{\alpha}\right)$ such that $A$ is topologically $*$ isomorphic to a closed self-adjoint subalgebra of $Q\left(\Pi H_{\alpha},\left\{\|\cdot\|_{\alpha}\right\}\right)$.

Proof. Given $\left(A,\left\{q_{\alpha}\right\}\right)$, consider the composition of the topological * isomorphism $a \rightarrow\left(a_{\alpha}\right)$ of $\left(A,\left\{q_{\alpha}\right\}\right)$ into the product of $B^{*}$-algebras $\Pi\left(\bar{A}_{\alpha},\|\cdot\|_{\alpha}\right)$, (Giles and Koehler (1973; page 88)), the topological * isomorphism $\left(a_{\alpha}\right) \rightarrow\left(T_{a}^{\alpha}\right)$ of $\Pi\left(\bar{A}_{\alpha},\|\cdot\|_{\alpha}\right)$ into $\Pi\left(B\left(H_{\alpha}\right),\|\cdot\|_{\alpha}\right)$ where for each $\alpha,\left(H_{\alpha},\|\cdot\|_{\alpha}\right)$ is the Hilbert space given by the Gelfand-Naimark Representation Theorem for the $B^{*}$. algebra $\left(\bar{A}_{\alpha},\|\cdot\|_{\alpha}\right)$, and the topological ${ }^{*}$ isomorphism $\left(T^{\alpha}\right) \rightarrow T$ of $\Pi\left(B\left(H_{\alpha}\right)\|\cdot\|_{\alpha}\right)$ into $Q\left(\Pi H_{\alpha},\left\{\|\cdot\|_{\alpha}\right\}\right)$ defined by $(T x)_{\alpha}=T^{\alpha} x_{\alpha}$ where the *-operation is defined on $Q\left(\Pi H_{\alpha},\left\{\|\cdot\|_{\alpha}\right\}\right)$ as in Theorem 7. It is clear that the image of $A$ in $Q\left(\Pi H_{\alpha},\left\{\|\cdot\|_{\alpha}\right\}\right)$ under this topological ${ }^{*}$ isomorphism is a self-adjoint subalgebra and since $A$ is complete this image is closed in $Q\left(\Pi H_{\alpha},\left\{\|\cdot\|_{\alpha}\right\}\right)$.

In a Hilbert space $H$, because of the convexity of the spatial numerical range we have that the spatial numerical range of an operator $T$ on $H$ and the algebra numerical range of the operator $T$ in $B(H)$ have the same closure. We now show that there is a similar relation between the spatial numerical range of a quotient bounded operator $T$ on a product of Hilbert spaces $\Pi\left(H_{\alpha}, \mid \cdot \|_{\alpha}\right)$ and the algebra numerical range of the operator $T$ in $Q\left(\Pi H_{\alpha},\left\{\|\cdot\|_{\alpha}\right\}\right)$.

THEOREM 9. Given a family $\left\{\left(H_{\alpha},\|\cdot\| \alpha\right)\right\}$ of Hilbert spaces, for any $T \in Q\left(\Pi H_{\alpha},\left\{\|\cdot\|_{\alpha}\right\}\right)$ we have

$$
\overline{V\left(\Pi H_{\alpha},\left\{\|\cdot\|_{\alpha}\right\} ; T\right)}=\overline{V\left(Q,\left\{q_{\alpha}\right\} ; T\right)} .
$$

Proof. Since we are dealing with Hilbert spaces we have for each $\alpha$,

So

$$
V\left(B\left(H_{\alpha}\right),\|\cdot\|_{\alpha} ; T^{\alpha}\right)=\overline{V\left(H_{\alpha},\|\cdot\|_{\alpha} ; T^{\alpha}\right)} .
$$

$$
\begin{aligned}
V\left(Q,\left\{q_{\alpha}\right\} ; T\right) & =\bigcup_{\alpha} V\left(B\left(H_{\alpha}\right),\|\cdot\|_{\alpha} ; T^{\alpha}\right) \\
& =\bigcup_{\alpha} \overline{V\left(H_{\alpha},\|\cdot\|_{\alpha} ; T^{\alpha}\right)} \subseteq \overline{\bigcup_{\alpha} V\left(H_{\alpha},\|\cdot\|_{\alpha} ; T^{\alpha}\right)} \\
& =\overline{V\left(\Pi H_{\alpha},\left\{\|\cdot\|_{\alpha}\right\} ; T\right)},
\end{aligned}
$$

and the result follows by Theorem 2 . 


\section{References}

F. F. Bonsall and J. Duncan (1971), Numerical range of operators on normed spaces and of elements of normed algebras, (Lond. Math. Soc. Lecture Note Series 2, Cambridge 1971).

F. F. Bonsall and J. Duncan (1973), 'Numerical ranges II' (Lond. Math. Soc. Lecture Note Series 10, Cambridge 1973).

J. R. Giles and D. O. Koehler (1973), 'On numerical ranges of elements of locally $m$-convex algebras', Pacific J. Math. 49, 79-91.

R. B. Holmes (1968), 'A formula for the spectral radius of an operator', Amer. Math. Monthly 75, $163-166$.

E. A. Michael (1952), 'Locally multiplicatively convex topological algebras', Mem. Amer. Math. Soc. 11 (1952).

R. T. Moore (1969), 'Banach algebras of operators on locally convex spaces', Bull. Amer. Math. Soc. $75,68-73$.

R. T. Moore (1969a), 'Adjoints, numerical ranges, and spectra of operators on locally convex spaces', Bull. Amer. Math. Soc. 75, 85-90.

The University of Newcastle, N.S.W.

Australia

Miami University, Oxford, Ohio

U.S.A.

The University of New England, N.S.W.

Australia 